\author{
Andreas Christe \\ Harriet Thoeny \\ Steffen Ross \\ Danny Spendlove \\ Dechen Tshering \\ Stephan Bolliger \\ Silke Grabherr \\ Michael J. Thali \\ Peter Vock \\ Lars Oesterhelweg
}

\section{Life-threatening versus non-life-threatening manual strangulation: are there appropriate criteria for MR imaging of the neck?}

Received: 4 November 2008

Revised: 8 January 2009

Accepted: 22 January 2009

Published online: 13 March 2009

(C) European Society of Radiology 2009

A. Christe $(\bowtie) \cdot H$. Thoeny •

D. Tshering $\cdot$ P. Vock

Department of Radiology, Inselspital,

University of Bern,

Freiburgstrasse,

3010 Bern, Switzerland

e-mail: andreas.christe@insel.ch

Tel.: +41-316-322111

A. Christe - S. Ross - D. Spendlove ·

S. Bolliger - M. J. Thali .

L. Oesterhelweg

Institute of Forensic Medicine,

University of Bern,

Buehlstrasse 20,

3012 Bern, Switzerland

\section{S. Grabherr}

Institute of Forensic Medicine,

University Hospital of Lausanne,

Rue du Bugnon 21,

1005 Lausanne, Switzerland

\author{
L. Oesterhelweg \\ State Institute of Legal Medicine \\ Berlin, Senate Administration for \\ Health, Environment and Consumer \\ Protection, \\ Turmstrasse 21, \\ 10559 Berlin, Germany
}

\begin{abstract}
The aim of the study was to determine objective radiological signs of danger to life in survivors of manual strangulation and to establish a radiological scoring system for the differentiation between life-threatening and non-life-threatening strangulation by dividing the cross section of the neck into three zones (superficial, middle and deep zone). Forensic pathologists classified 56 survivors of strangulation into life-threatening and non-lifethreatening cases by history and clinical examination alone, and two blinded radiologists evaluated the MRIs of the neck. In 15 cases, stran-
\end{abstract}

gulation was life-threatening (27\%), compared with 41 cases in which strangulation was non-life-threatening $(73 \%)$. The best radiological signs on MRI to differentiate between the two groups were intramuscular haemorrhage/oedema, swelling of platysma and intracutaneous bleeding (all $\mathrm{p}=0.02$ ) followed by subcutaneous bleeding $(\mathrm{p}=0.034)$ and haemorrhagic lymph nodes $(\mathrm{p}=0.04)$, all indicating life-threatening strangulation. The radiological scoring system showed a sensitivity and specificity of $\approx 70 \%$ for life-threatening strangulation, when at least two neck zones were affected. MRI is not only helpful in assessing the severity of strangulation, but is also an excellent documentation tool that is even admissible in court.

Keywords Manual strangulation MRI of the neck - Radiological zone score for life-threatening strangulation

\section{Introduction}

Strangulation is a generic term for hanging, ligature and manual strangulation. Pathophysiologically, there are three mechanisms of strangulation leading to death [1-3]: (1) venous obstruction, leading to cerebral stagnation, hypoxia, and unconsciousness, which allows muscle tone relaxation and final arterial and airway obstruction; (2) arterial spasm due to carotid pressure, leading to low cerebral blood flow and collapse; (3) vagal collapse caused by pressure to the carotid sinuses and increased parasympathetic tone. Interestingly, none of the proposed mechanisms emphasises airway compromise alone. In fact, although delayed mechanical airway compromise occurs and often complicates patient management, it appears to play a minor role in the immediate death of victims of fatal strangulation. Regardless of disagreement on theories, most experts agree that death ultimately occurs from cerebral hypoxia and ischaemic neuronal death.

While hanging is generally applied with suicidal intention, manual choking and ligature strangulation are mostly acts of violence. It is not possible to commit suicide by manual strangulation, because once consciousness is lost, the muscle tone in the strangulating arms relaxes.

Strangulation is an important medico-legal issue and challenge, especially with survivors of manual strangula- 
tion. The most important and most difficult decision to be made is whether or not life was in danger, because the allor-nothing law-death or complete recovery-is mostly present. Therefore, forensic pathologists have to interview and examine the victims thoroughly and as fast as possible after the incident. Signs of life-threatening strangulation are sight impairment, loss of consciousness, loss of urine or ordure, petechial conjunctival bleeding from blood congestion and strangulation marks [4]. However, it is usually very difficult because forensic pathologists need objective facts and not uncertain case histories. Computed tomography (CT) and magnetic resonance imaging (MRI) of the neck [5] provide more objective data. In clinical radiology, there is CT and MRI experience with neck injuries in survivors of strangulation. CT examinations of the neck and throat in cases of hanging have been described, mainly in case reports [6-10]. MRI allows for better assessment of the inner structures of the neck, like soft tissue, vessels and larynx that cannot be clinically examined or documented. Yen et al. [11] investigated 14 survivors of manual strangulation with MRI and found typical strangulation signs such as subcutaneous and intramuscular haemorrhage (platysma), haemorrhage of lymph nodes and salivary glands, but few injuries in the larynx and hyoid, which is more typical on post-mortem MRI, CT or autopsy. To our knowledge, no radiological sign was ever classified as lifethreatening in survivors of strangulation. The aim of this study is to identify all pathological features on MRI arising from manual strangulation, to statistically find radiological signs on MRI of life-threatening strangulation and to discuss these findings together with typical autopsy findings of victims who died (gold standard). Furthermore, the correlation between MRI features and each clinical finding shall be statistically examined.

\section{Materials and methods}

\section{Patient characteristics}

We examined 56 survivors of strangulation incidents (37 strangulations with hands, 6 ligature strangulations, 5 choke holds and 8 mixed strangulations), consisting of 35 women and 21 men with a median age of 26.5 years, range 12 54 years. These cases represented a continuous sample of victims who were admitted to our institute by the local justice department for forensic examination, documentation and reconstructions of the sequence of events. This study was approved by the local ethics committee, and written informed consent was obtained from all study participants.

\section{Forensic examination}

A board-certified forensic pathologist performed the standard forensic examination a few hours after the incident, except for one victim who did not report the incidence for $432 \mathrm{~h}$. The median delay between strangulation and forensic examination was $9.5 \mathrm{~h}$ (mean $20.4 \mathrm{~h}$ ). Two board-certified forensic pathologists in consensus made the assessment of danger to life. The following objective signs of strangulation were assessed: congestive ophthalmic petechial haemorrhages, strangulation marks, haematoma, erythema, skinabrasion and swelling of the neck. Subjective signs of strangulation like loss of consciousness, hallucinatory phenomena, loss of urine or faeces, dysphagia, dyspnoea, sore throat and croakiness were specifically queried. A case was classified as life-threatening when either congestive ophthalmic petechial haemorrhages or substantial objective and subjective signs of strangulation were present.

\section{Magnetic resonance imaging}

MRI was performed with a median delay of $38 \mathrm{~h}$ (mean $50 \mathrm{~h}$ ) to strangulation. After excluding contra-indications, the victims gave their written informed consent to perform MRI of the neck. A 1.5-T MRI Unit (Magnetom Sonata, Siemens, Erlangen, Germany) with a neck coil (CP Neck Array) was used for imaging. The MRI neck protocol included six sequences without oral or intravenous contrast:

(1) Coronary TIRM sequence (turbo-inversion-recoverymagnitude): repetition time (TR) $5,620 \mathrm{~ms}$, echo time (TE) $28 \mathrm{~ms}$, slice thickness $4 \mathrm{~mm}$, distance factor $30 \%$, field of view (FoV) $340 \mathrm{~mm}$, resolution 512, voxel size $1.3 \times 0.7 \times 4.0 \mathrm{~mm}$.

(2) Axial TIRM sequence: TR 4,000 ms, TE $20 \mathrm{~ms}$, slice thickness $3 \mathrm{~mm}$, distance factor $20 \%$, FoV $280 \mathrm{~mm}$, resolution 512, voxel size $1.1 \times 0.5 \times 3.0 \mathrm{~mm}$.

(3) Axial T2-weighted TSE sequence (turbo-spin-echo): TR 3,930 ms, TE $76 \mathrm{~ms}$, slice thickness $3 \mathrm{~mm}$, distance factor $20 \%$, FoV $280 \mathrm{~mm}$, resolution 512, voxel size $1.1 \times 0.5 \times 3.0 \mathrm{~mm}$.

(4) Axial T1-weighted TSE: TR $687 \mathrm{~ms}$, TE $12 \mathrm{~ms}$, slice thickness $3 \mathrm{~mm}$, distance factor $20 \%$, FoV $280 \mathrm{~mm}$, resolution 512 , voxel size $1.1 \times 0.5 \times 3.0 \mathrm{~mm}$.

(5) Axial T2 trueFISP sequence (true fast imaging with steady-state precession): TR $4.48 \mathrm{~ms}$, TE $2.24 \mathrm{~ms}$, slice thickness $3 \mathrm{~mm}$, distance factor $20 \%$, FoV $200 \mathrm{~mm}$, resolution 256 , voxel size $0.8 \times 0.8 \times 3 \mathrm{~mm}$.

(6) Axial T1 TSE fat-saturated sequence: TR $693 \mathrm{~ms}$, TE $12 \mathrm{~ms}$, slice thickness $4 \mathrm{~mm}$, distance factor $20 \%$, FoV $300 \mathrm{~mm}$, resolution 512 , voxel size $1.2 \times 0.6 \times 4.0 \mathrm{~mm}$.

The total MR neck examination time did not exceed $1 \mathrm{~h}$.

MR image analysis

Two board-certified radiologists (sub-specialised in head and neck radiology) evaluated the MR images on a PACS 
workstation (picture archiving and communication system) in independent, blinded readings. In accordance with typical autopsy findings that are most frequently found in cases of hanging or severe manual strangulation [12-15], muscles of the neck (mainly the sternocleidomastoid, strap and platysma muscles), subcutaneous tissue, larynx, pharynx, hyoid, salivary glands, thyroid and lymph nodes were thoroughly analysed for oedema or haemorrhage, and the vessels were evaluated for integrity. It is often not possible to differentiate blood from oedema on MRI [16, 17], and as a co-appearance of both is frequent in traumatised tissue, these signs were classified together. Intra-, subcutaneous, intramuscular, perilaryngeal and parapharyngeal oedema or haemorrhage were defined as diffuse areas of TIRM or T2 hyperintensity and T1 hypo- or hyperintensity. Special attention was paid to the platysma, since involvement of the platysma was suspected to be a specific sign of strangulation by Yen et al. [5, 11], but the sternocleidomastoid, strap and dorsal neck muscles were assessed together. The platysma muscle was considered affected when it was thickened or hyperintense on the TIRM or T2weighted sequence. A hyperintense appearance of the vocal cords on the TIRM sequence was rated as glottic oedema. Salivary glands, thyroid gland and lymph nodes were considered positive for haemorrhage or oedema when a parenchymal TIRM/T2 hyperintensity and T1 hypo-/ hyperintensity appeared. Vessel rupture was defined as T1 iso-/hyperintense and T2/TIRM hyperintense blood around the jugular vein or the carotid artery with mass effect.
Intramural haemorrhage should show a hyperintense crescent-shaped widening of the carotid artery wall in the T1-weighted fat-saturated sequence, and dissection of the carotid artery must demonstrate a hypointense dissection membrane in the arterial lumen on trueFISP images. Laryngeal cartilage and hyoid bone were rated as fractured when a loss of integrity or a TIRM hyperintense and T1 hypointense fracture line was obvious. Additionally, lymph nodes were considered enlarged, when the long axis measured more than $12 \mathrm{~mm}$ and the short axis more than $10 \mathrm{~mm}$. To be judged to be pathological, alterations had to be identified on more than one MR sequence and plane each. In view of the potential juridical consequences of forensic diagnosis, only the findings that were detected by both radiologists were considered positive. Inter-rater agreement was calculated.

We divided the cross section of the neck into three zones for establishing a radiological score and for a better understanding of the danger of strangulation (Fig. 4). Superficial zone A includes skin and subcutaneous fatty tissue. Middle zone B contains muscles, vessels, salivary glands and cervical lymph nodes. Deep zone $\mathrm{C}$ includes larynx and perilaryngeal tissue. A radiological score was designed from 0 to 3 depending on the amount of zones affected (Fig. 4); 0 stood for no radiological finding. A score of 3 meant a pathological MRI finding in all three zones with at least one pathological feature per zone. A score of 2 indicated 2 pathologic zones, and a score of 1 meant that only 1 zone was affected.

Table 1 MRI findings and case data of 56 strangulation survivors divided in life-threatening and not life-threatening strangulation. Signs with significant $\mathrm{p}$-values for best differentiation between the two groups are showed in bold face. The Kappa-test was applied for inter-rater agreement of the two radiologists

\begin{tabular}{|c|c|c|c|c|c|c|c|c|c|}
\hline \multicolumn{3}{|c|}{ STRANGULATION } & \multicolumn{2}{|c|}{$\begin{array}{r}\text { total strangulation } \\
\qquad \mathrm{n}=56\end{array}$} & \multicolumn{2}{|c|}{$\begin{array}{r}\text { no danger to life } \\
n=41\end{array}$} & \multicolumn{2}{|c|}{$\begin{array}{r}\text { danger to life } \\
n=15\end{array}$} & \multirow{2}{*}{$\begin{array}{l}\text { p-value fischer exact: } \\
\text { differentiation between life } \\
\text { threatening and non life } \\
\text { threatening strangulation } \\
\mathbf{0 . 0 2}\end{array}$} \\
\hline \multirow{18}{*}{ 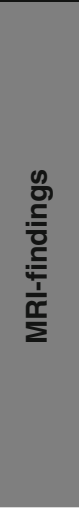 } & \multirow{2}{*}{$\begin{array}{l}\text { soft tissue } \\
\text { (superficial) }\end{array}$} & intracutaneous hemorrhage / edema ${ }^{1}$ & 16 & $29 \%$ & 8 & $20 \%$ & 8 & $53 \%$ & \\
\hline & & subcutaneous hemorrhage / edema ${ }^{2}$ & 31 & $55 \%$ & 19 & $46 \%$ & 12 & $80 \%$ & 0.034 \\
\hline & \multirow{2}{*}{$\begin{array}{l}\text { soft tissue } \\
\text { (deep) }\end{array}$} & intramuscular hemorrhage / edema ${ }^{3}$ & 16 & $29 \%$ & 8 & $20 \%$ & 8 & $53 \%$ & 0.02 \\
\hline & & swelling of platysma ${ }^{2}$ & 16 & $29 \%$ & 8 & $20 \%$ & 8 & $53 \%$ & 0.02 \\
\hline & \multirow{3}{*}{ larynx } & glottic edema ${ }^{2}$ & 5 & $9 \%$ & 2 & $5 \%$ & 3 & $20 \%$ & 0.113 \\
\hline & & perilaryngeal bleeding / edema ${ }^{2}$ & 8 & $14 \%$ & 4 & $10 \%$ & 4 & $27 \%$ & 0.19 \\
\hline & & cartilage fracture & 0 & $0 \%$ & 0 & $0 \%$ & 0 & $0 \%$ & 1 \\
\hline & pharynx & parapharyngeal hemorrhage / edema ${ }^{2}$ & 7 & $13 \%$ & 3 & $7 \%$ & 4 & $27 \%$ & 0.0743 \\
\hline & hyoid & hyoid fracture & 0 & $0 \%$ & 0 & $0 \%$ & 0 & $0 \%$ & 1 \\
\hline & \multirow{2}{*}{ salivary glands } & submandibular hemorrhage / edema ${ }^{2}$ & 4 & $7 \%$ & 1 & $2 \%$ & 3 & $20 \%$ & 0.0545 \\
\hline & & parotid hemorrhage / edema ${ }^{2}$ & 4 & $7 \%$ & 2 & $5 \%$ & 2 & $13 \%$ & 0.289 \\
\hline & thyroid gland & thyroid hemorrhage / edema ${ }^{3}$ & 2 & $4 \%$ & 1 & $2 \%$ & 1 & $7 \%$ & 0.468 \\
\hline & \multirow{3}{*}{ vessels } & rupture & 0 & $0 \%$ & 0 & $0 \%$ & 0 & $0 \%$ & 1 \\
\hline & & intramural hemorrhage & 0 & $0 \%$ & 0 & $0 \%$ & 0 & $0 \%$ & 1 \\
\hline & & dissection & 0 & $0 \%$ & 0 & $0 \%$ & 0 & $0 \%$ & 1 \\
\hline & \multirow{2}{*}{ Iymph nodes } & hemorrhage / edema ${ }^{3}$ & 12 & $21 \%$ & 6 & $15 \%$ & 6 & $40 \%$ & 0.04 \\
\hline & & enlargement $t^{3}$ & 16 & $29 \%$ & 14 & $34 \%$ & 2 & $13 \%$ & 0.186 \\
\hline & MRI-Score & 0-3 zones & & 1.09 & & 0.85 & & 1.73 & $0.016^{w}$ \\
\hline \multirow{5}{*}{ 悉 } & \multicolumn{2}{|c|}{ age (mean/median) } & 28.7 & 26.5 & 29.0 & 28.0 & 27.9 & 26.0 & $0.684^{W}$ \\
\hline & \multicolumn{2}{|l|}{$\operatorname{sex} m$} & 21 & $38 \%$ & 16 & $39 \%$ & 5 & $33 \%$ & 0.764 \\
\hline & \multicolumn{2}{|l|}{$\operatorname{sex} f$} & 35 & $63 \%$ & 25 & $61 \%$ & 10 & $67 \%$ & 0.764 \\
\hline & \multicolumn{2}{|c|}{ delay between strangulation and clinical examination ( $h$, mean/median) } & 20.4 & 9.5 & 23.1 & 8.0 & 13.3 & 10.0 & $0.718^{W}$ \\
\hline & \multicolumn{2}{|c|}{ delay between strangulation and MRI ( $h$, mean/median) } & 50.0 & 38.0 & 55.8 & 40.0 & 33.9 & 37.0 & $0.267^{W}$ \\
\hline \multicolumn{3}{|c|}{$\mathrm{W}=$ Wilcoxon test } & \multicolumn{7}{|l|}{ Kappa test } \\
\hline \multicolumn{2}{|c|}{ significant } & $0.0000-0.0455$ & $1=$ fair agre & & \multicolumn{2}{|c|}{$0.2-0.4$} & & & \\
\hline \multicolumn{2}{|c|}{ indicative } & $0.0455-0.3173$ & ${ }^{2}=$ modera & reement & \multicolumn{2}{|c|}{$0.4-0.6$} & & & \\
\hline \multicolumn{2}{|c|}{ not significant } & $0.3173-1.0000$ & ${ }^{3}=$ substan & greement & \multicolumn{2}{|c|}{$0.6-0.8$} & & & \\
\hline
\end{tabular}




\section{Statistics}

Radiological signs, sex ratio and strangulation type in the two groups - danger and no danger to life-were compared using Fisher's exact test. The radiological score, age and delay between examinations of the two groups were statistically analysed by the Wilcoxon rank sum test. A sign with a $\mathrm{p}$ value of less than 0.0455 [twice the standard deviation (SD)] was considered significant for danger to life. Indicative of danger to life was a $p$ value between 0.0455 and $0.3173(\mathrm{SD})$. $\mathrm{P}$ values over 0.3173 were rated as not significant. Sensitivity and specificity for lifethreatening strangulation were calculated for each radiological score and the receiver-operating characteristic (ROC) was computed for best cutoff scores. To establish the inter-rater agreement of the two radiologists, the weighted kappa coefficient $[18,19]$ was calculated. The association between the clinical and MRI findings was calculated with the chi-square test.

\section{Results}

\section{Forensic findings}

The forensic pathologists classified $27 \%(15 / 56)$ of the strangulation cases as life-threatening and $73 \%(41 / 56)$ as non-life-threatening.

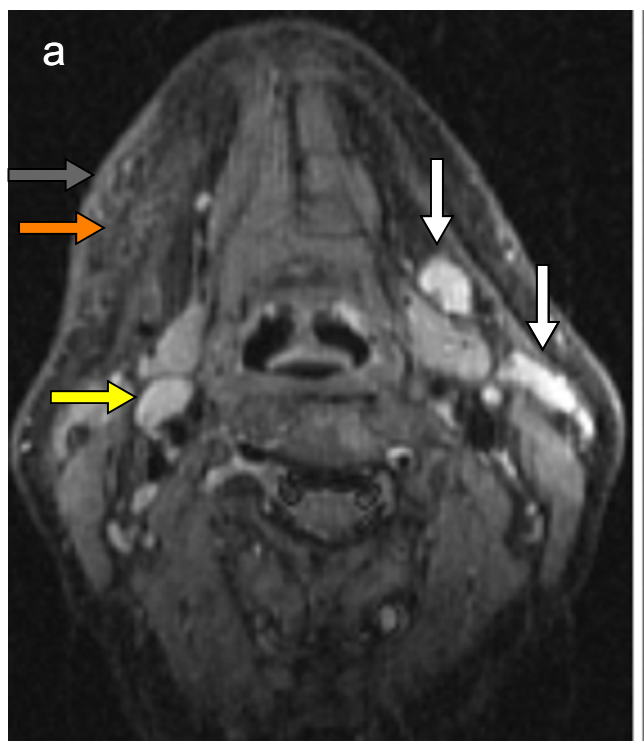

Fig. 1 MRI of a 45-year-old female victim $4 \mathrm{~h}$ after strangulation displaying lymph node haemorrhage and oedema, intra- and subcutaneous haemorrhage. a Axial fat-saturated T2-weighted sequence (TIRM) shows two very hyperintense lymph nodes submandibular on the left (white arrows), compared to the reference node submandibular on the right (yellow arrow). b On the T1weighted sequence on the same level, the oedematous node is
Case information

Although the sex ratio was in favour of women in the danger to life group $(67 \% ; 10 / 15)$, compared with the no danger to life group $(61 \% ; 25 / 41)$, this was not significant ( $p$ value 0.76 ), meaning that an equal number of men suffered lifethreatening strangulation relatively. Neither age nor delay between strangulation and examinations helped to divide the two groups ( $\mathrm{p}$ values $0.27-0.72$ ). The case information and radiological findings are listed in Table 1.

\section{MRI findings}

Soft tissue was the most severely affected part of the neck in all strangulation cases (Figs. 1, 2 and 3). Mainly subcutaneous haemorrhage/oedema was observed in 55\% (31/56), followed by intracutaneous haemorrhage and intramuscular haemorrhage; both appeared in $29 \%$ $(16 / 56)$. Swelling of platysma was also detected in $29 \%$ (16/56) of the cases (Fig. 2). These findings were seen significantly more often in the danger to life group ( $p$ values between 0.02 and 0.034$)$ : $80 \%(12 / 15)$ of the lifethreatened group showed subcutaneous haemorrhage versus $46 \%(19 / 41)$ of the non-life-threatened group. Fifty-three percent $(8 / 15)$ of the victims of the danger to life group had intramuscular bleeding/oedema, while only $20 \%$ of the non-endangered group showed the same

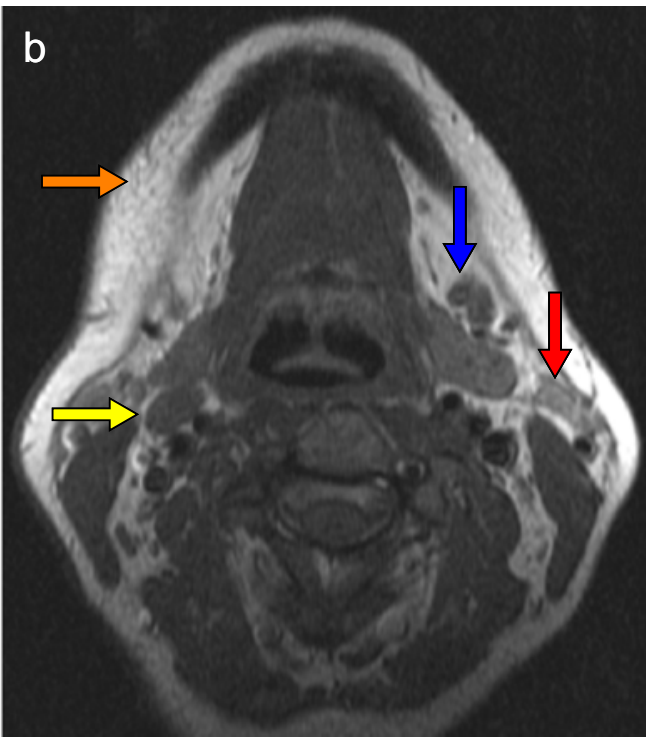

slightly hypointense (blue arrow), while the haemorrhagic node is hyperintense (red arrow) compared to the normal node on the right (yellow arrow). Note the intracutaneous and subcutaneous haemorrhage, better detectable on the TIRM sequence as hyperintense skin thickening (a, grey arrow) and hyperintense imbibition of the subcutaneous tissue. Subcutaneous haemorrhage is hypointense in T1-weighted sequence (b, orange arrow) 


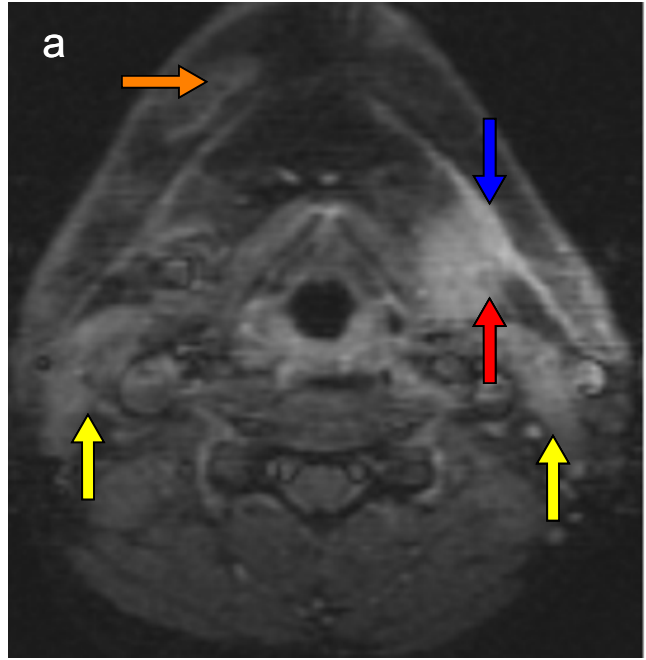

Fig. 2 Twenty hours after strangulation, the MRI of this 36-year-old female patient shows haemorrhagic swelling of platysma, haemorrhage around the submandibular gland and subcutaneous haemorrhage. a: Axial fat-saturated T2-weighted sequence (TIRM): hyperintense swelling of platysma (blue arrow) with adjacent haemorrhage into fatty tissue alongside the submandibular gland

pathological feature. Intracutaneous haemorrhage and swelling of the platysma were demonstrated in the same number of patients in the two groups, as last mentioned above (Table 1).

Haemorrhagic lymph nodes (Fig. 1) appeared overall in every fifth victim and also proved to be a good discriminator for life-threatening strangulation ( $\mathrm{p}$ value 0.04 ), but enlargement of lymph nodes was more often seen in non-endangered victims. Interestingly, the larynx was less frequently involved: perilaryngeal bleeding (Fig. 3) occurred overall in 14\% (8/56) and haemorrhage/ oedema of the vocal cord only in $9 \%(5 / 56)$; against all expectations, both were not significant for, but only indicative of danger to life ( $p$ value between $0.1-0.2$ ). The submandibular and parotid glands were both involved in only 7\% (4/56) of haemorrhages, although submandibular haemorrhage was strongly indicative of danger to life $(\mathrm{p}=0.055)$. In $4 \%(2 / 56)$ the thyroid gland showed haemorrhage independently of danger to life. No fractures of the laryngeal skeleton, the hyoid bone or a vessel disruption occurred in this study of survivors.

Twenty-five victims had a normal MRI (apart from enlarged lymph nodes, which was the only radiological sign, and this correlated positively with no danger to life). Three of them were judged to be life-threatened by the forensic pathologists, based on congestive petechial haemorrhage $(n=2)$ or loss of consciousness $(n=1)$. In any case, a lack of radiological findings was a significant sign of no danger to life $(p=0.04)$. Sensitivity of a positive MRI (any of the above-mentioned findings, except enlarged lymph nodes) for life-threatening strangulation in this setting was 0.80 and specificity was 0.54 .

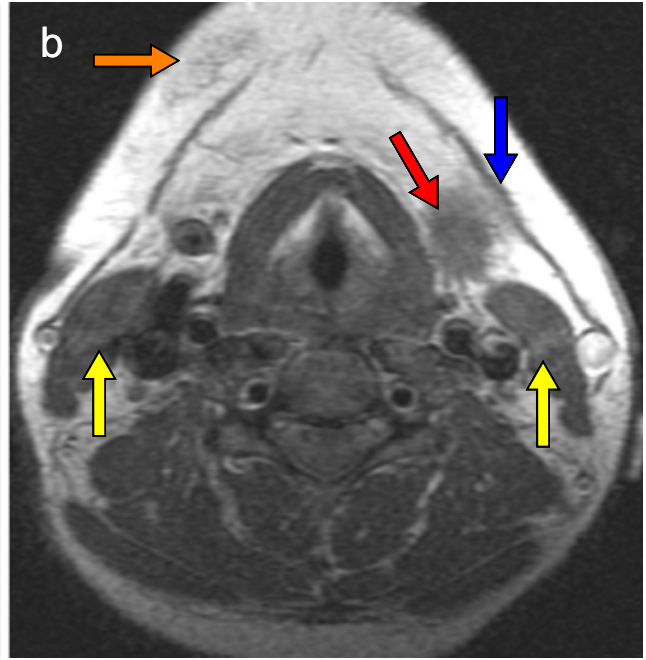

(red arrow). Subcutaneous oedema or haemorrhage occurs in the submental region (orange arrow). Note the haemorrhage in the sternocleidomastoid muscles (yellow arrows). b: On the T1weighted image, the border of the submandibular gland can more easily be depicted (red arrow)

Inter-rater agreement was highest for intramuscular haemorrhage/oedema with a weighted kappa coefficient $\mathrm{K}$ of $0.794(\mathrm{SD}=0.075)$, followed by haemorrhagic lymph nodes $(\kappa=0.717, \mathrm{SD}=0.087)$, indicating substantial agreement for both (Table 1). Only fair agreement was found for intracutaneous haemorrhage/oedema $(\kappa=0.349, \quad \mathrm{SD}=$ 0.153 ); for all other signs the radiologists showed moderate agreement $(\kappa=0.4-0.6)$.

Applying the designed radiological zone score (Fig. 4), there was a significant difference in the two groups ( $p$ value $=0.016$ ). A victim of the danger-to-life group showed on average 1.73 affected neck zones, and in a non-lifethreatened victim only 0.85 zones were afflicted.

Whenever a deeper radiological zone was affected (Fig. 4), all more superficial zones also showed a bleeding at the same or different location without exception. If for instance an oedema of the vocal cord appeared in zone $\mathrm{C}$, then zones B and A were always affected, for example, with an intramuscular and subcutaneous bleeding. When a victim showed a bleeding in the sternocleidomastoid muscle (zone B), there was always a cutaneous or subcutaneous haemorrhage (zone A). The risk of having suffered danger to life was dependent on the deepest zone affected (Fig. 4). The positive predictive value (= risk) for danger to life of a bleeding in zone A was $20 \%$, in zone B $50 \%$ and in zone $\mathrm{C} 44 \%$. The negative predictive value was $88 \%$ for negative MRI.

Sensitivity and specificity of MRI for life-threatening strangulation varied depending on the radiological score. When all three zones were affected, the score was 3 , and sensitivity for danger to life was $27 \%$ and specificity $88 \%$. For two or more zones affected (score $\geq 2$ ), sensitivity 

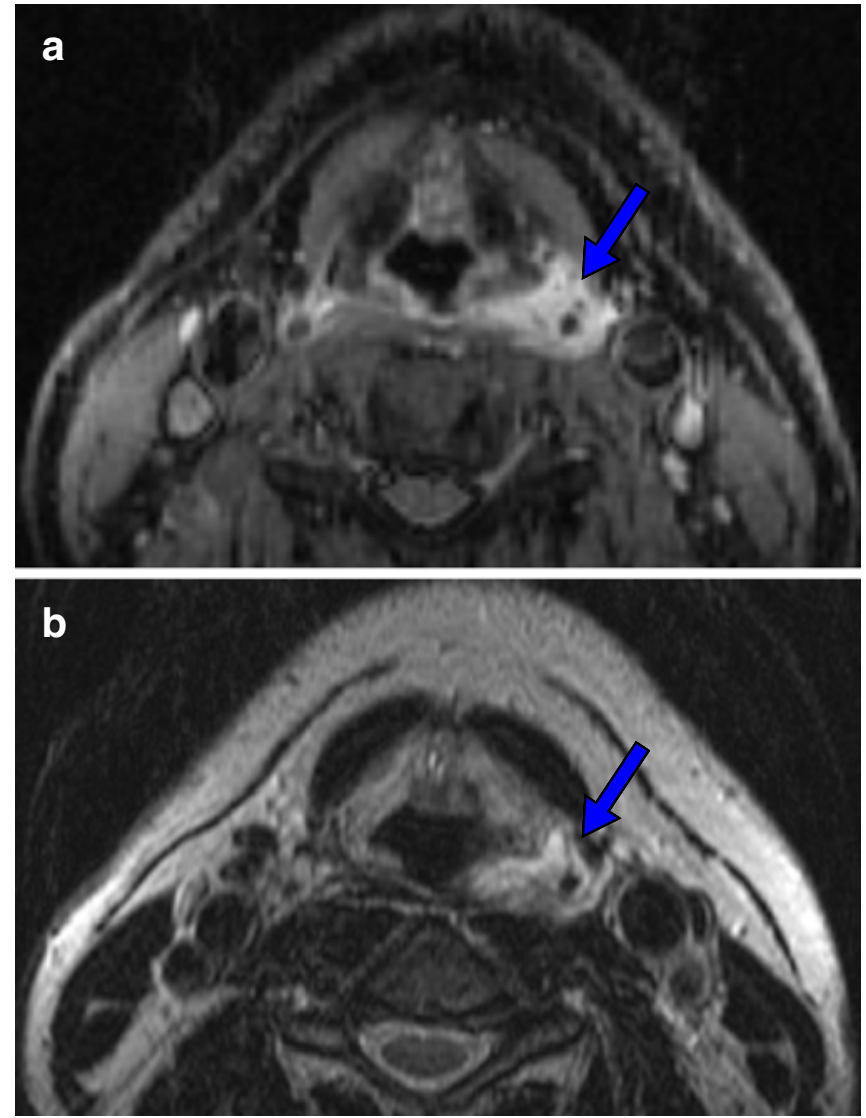

Fig. 3 MRI of a 36-year-old male victim, $24 \mathrm{~h}$ after manual strangulation with perilaryngeal bleeding. Hyperintense bleeding (blue arrow) around the hypointense superior horn of thyroid cartilage in fat-saturated T2-weighted (a) and T2-weighted sequence (b)

increased to $67 \%$ and specificity dropped to $73 \%$. If one or more zones were affected (score $\geq 1$ ), sensitivity for danger of life was $80 \%$, and specificity only $54 \%$. The receiveroperating characteristic (ROC) showed the best discrimination for a score $\geq 2$ (Fig. 5).

Association between clinical and MRI findings (independent of danger to life)

The nine patients who suffered from loss of consciousness showed an association with the following MRI findings: six had swelling of the platysma $(p=0.0183)$, four were depicted with perilaryngeal bleeding $(\mathrm{p}=0.0213)$ and four with peripharyngeal bleeding $(p=0.009)$; three showed oedema of the glottis $(p=0.03)$. Furthermore, loss of consciousness is significantly related to haemorrhage in the submandibular gland $(\mathrm{p}=0.0089)$ and thyroid gland $(\mathrm{p}=$ 0.021 ). A total of 25 cases with dyspnoea were found; 9 of them had an associated lymph node haemorrhage $(\mathrm{p}=$ $0.0395)$. Moderate association $(\mathrm{p}>0.05$ and $\mathrm{p}<0.3)$ was found between dysphagia and intramuscular haemorrhage; sore throat and perilaryngeal bleeding; ophthalmic petechial haemorrhages and intramuscular haemorrhage; haematoma of the neck and subcutaneous bleeding; erythema of the neck and intracutaneous bleeding.

\section{Discussion}

Until now, there have been no objective criteria for diagnosing life-threatening strangulation by imaging. Typical signs of strangulation described in previous studies [5, 11] have been confirmed; furthermore, typical radiological signs of life-threatening strangulation have been established. All known autopsy findings (gold standard) of the neck of strangulation victims who died [12-15] were studied in MRI. Apparently, some of these findings, such as vessel disruption and laryngeal fracture, did not occur and could be classified as deadly signs, but further studies are needed. On the other hand, haemorrhage into the submandibular, parotid

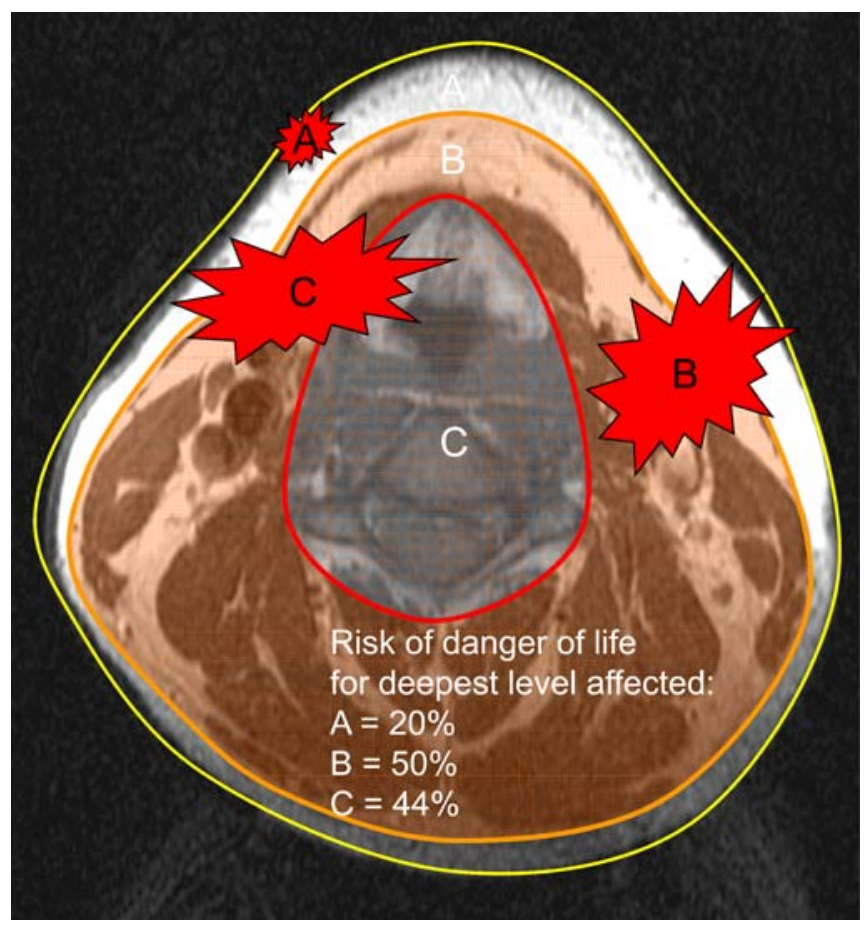

Fig. 4 The radiological score ranged from 0 to 3 depending on the number of zones affected. Superficial zone A included skin and subcutaneous fatty tissue. Zone B (danger zone) contained all between $\mathrm{A}$ and $\mathrm{C}$ : muscles, vessels, salivary glands and cervical lymph nodes. Deep zone C comprised larynx and perilaryngeal tissue. A score of 3 meant a pathologic finding in MRI in all three zones (C). Whenever a deeper radiological zone was affected, all more superficial zones showed also a bleeding at the same or another location without exception. The positive predictive value (= risk) for danger of life of a bleeding in zone A was $20 \%$, in zone B $50 \%$ and in zone C $44 \%$. The negative predictive value was $88 \%$ for a negative MRI 


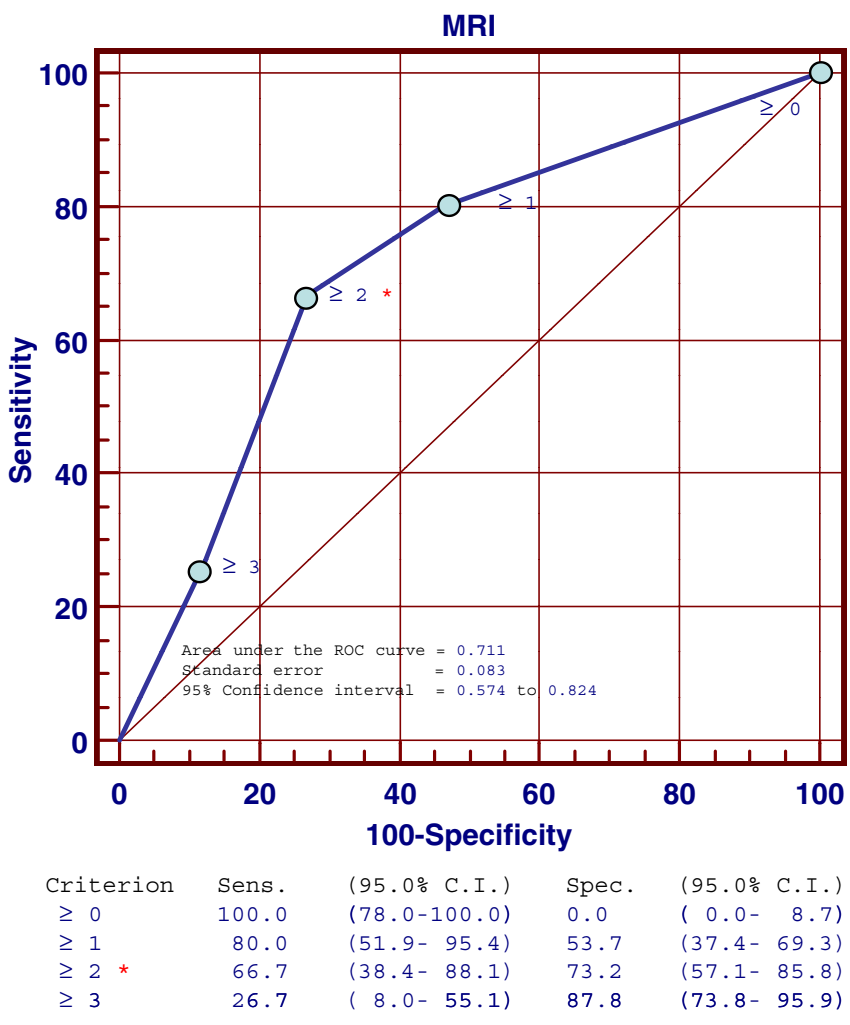

Fig. 5 Receiver-operating characteristic (ROC) of MRI score for detecting life-threatening strangulation. Area under the ROC curve was 0.771 . Best sensitivity and specificity are provided by a MRI score $\geq 2$

or thyroid gland — all signs of strangulation at autopsy — did not prove to be signs of danger to life in survivors. Victims who were forensically considered to have suffered danger to life showed significantly more findings in the superficial and middle zones (zones A and B), such as intracutaneous, subcutaneous and intramuscular haemorrhage/oedema, significantly more swelling of the platysma and haemorrhagic lymph nodes of the neck. Against all expectations, the deep pathologies in zone $\mathrm{C}$ like glottic oedema, perilaryngeal or parapharyngeal haemorrhage did not differ significantly in the two groups. The (peri-) laryngeal haemorrhage could arise more from the frustrating attempt of breathing or from the defending mechanisms to keep the airways open.

A radiological zone score proved to be useful: sensitivity and specificity for life-threatening strangulation were approximately $70 \%$. Interestingly, all more superficial radiological zones were affected when a deeper finding occurred, supporting the theory that there could be a linear or gradual disposal of energy from the skin to the larynx or spine during the act of strangulation.

Haemorrhage nearby the carotid artery and the jugular vein (zone B), like intramuscular bleeding, correlated better with danger to life than injuries to the upper airways, supporting the theory that the immediate cause of death is more the lowered blood supply to the brain than the low air supply to the lung. Therefore, zone B could also be referred to as 'danger zone'. Maybe the non-life-threatened victims have the ability to shunt some of the energy away from the danger zone $\mathrm{B}$ to the deepest zone $\mathrm{C}$ to avoid compression or damage to the vessels. On the other hand, all congestive petechial bleedings are judged life threatening by forensic pathologists; therefore, it is not surprising that alterations around vessels and carotid sinuses in MRI correlate better with danger of life than airway alterations.

As already mentioned in the introduction, venous obstruction is a leading cause of death in strangulation and mainly responsible for the ophthalmic congestive petechial haemorrhages. It seems that the haemorrhagic lymph nodes are the inner correlate to these superficial bleedings, because the bleeding mechanism is the same and the finding correlates significantly to danger of life also. Therefore, special attention to this finding should be given in the assessment of danger to life, and further strangulation studies should focus on it. Lymph node enlargement is statistically indicative of a non-life-threatening strangulation. In other terms, none of the haemorrhagic lymph nodes were enlarged, and invulnerable victims showed larger nodes. Furthermore, younger people - like most of our study population-have larger lymph nodes than the elderly.

Not every T2-hyperintensity in the skin or in subcutaneous tissue should be considered as a result of strangulation. Acne as well can show the same T1-hypo- and T2-hyperintense alterations, but displays smaller, more focal or multifocal, localised, patchy and not diffuse changes.

Looking at the correlation between clinical and MRI findings (independent of danger to life), it is not surprising that loss of consciousness showed the most significant associations with the mentioned MRI findings like swelling of the platysma, oedema of the glottis and submandibular gland haemorrhage, although the latter two could not be significantly linked to danger to life. Moderate association could be found for corresponding clinical and MRI findings such as dysphagia and intramuscular bleeding, sore throat and perilaryngeal bleeding or haematoma of the neck and subcutaneous haemorrhage. But surprisingly there was no association between croakiness and oedema of the glottis or between skin abrasion and intracutaneous bleeding. Maybe these clinical symptoms were just too small for any corresponding MRI feature to be depicted.

\section{Shortcomings}

Because of a small number of patients, no fracture of the laryngeal skeleton, hyoid bone or vessel disruption occurred in this study sample. These lesions probably result from a more powerful (hanging) or violent act of strangulation, where the victims frequently die. If one of those lesions should appear in MRI, life was probably in danger, but further studies of survivors of strangulation need to be done. 
The appropriateness of life-threatening strangulation is crucial for forensic pathologists, because the degree of penalty for the offender in court only depends on this decision alone. For a forensic pathologist the assessment of strangulation is very difficult with a subjective case history only, often without witness and only one objective clinical examination, without knowing the morphology of the deeper neck. Therefore, MRI provides a second objective proof of trauma, helping forensic pathologists to judge much better the severity of strangulation. In this way, radiologists can be more and more aware of the typical signs of life-threatening manual strangulation.
Last but not least, MRI is an excellent general documentation tool, providing the specialist and the layman a comprehensive overview of complex neck injuries $[20,21]$.

Acknowledgements The authors are grateful to the entire Virtopsy team and their research partners (see www.virtopsy.com), who made this study possible due to their exceptional commitment. Special thanks go to the forensic pathologists of the Institute of Forensic Medicine (University of Bern, Switzerland), for their expertise and support during the study. Futhermore, we thank the team of highly motivated radiology technicians of the MRI-division of the Department of Radiology at Inselspital, (University of Bern, Switzerland).

\section{References}

1. Wahlen BM, Thierbach AR (2002) Near-hanging. Europ J Emerg Med 9:348-350, etc

2. McHugh TP, Stout M (1983) Nearhanging injury. Ann Emerg Med 12:774-776

3. Iserson KV (1984) Strangulation: a review of ligature, manual, and postural neck compression injuries. Ann Emerg Med 13:179-185

4. Plattner T, Bolliger S, Zollinger U (2005) Forensic assessemet of survived strangulation. Forensic Sci Int 153(23):202-207

5. Yen K, Thali MJ, Aghayev E, Jackowski C, Schweitzer W, Boesch C, Vock P, Dirnhofer R, Sonnenschein M (2005) Strangulation signs: initial correlation of MRI, MSCT, and forensic neck findings. J Magn Reson Imaging 22(4):501-510

6. Linnau KF, Cohen WA (2002) Radiologic evaluation of attempted suicide by hanging: cricotracheal separation and common carotid artery dissection. AJR AM J Roentgenol 178-214

7. Poquet E, Dibiane A, Jourdain C, el Amine M, Jacob A, Escure MN (1995) Blunt injury of the larynx by hanging. X-ray computed tomographic aspect (in French). J Radiol 76:107-109

8. Noguchi K, Matsuoka Y, Hohda K, Katsuyama J, Nishimura S (1992) A case of common carotid artery stenosis due to hanging (in Japanese). No Shinkei Geka 20:1185-1188
9. Lupetin AR, Hollander M, Rao VM (1998) CT evaluation of laryngotracheal trauma. Semin Musculoskel Radio 2:105-116

10. Meglin AJ, Biedlingsmaier JF, Mirvis SE (1991) Three-dimensional computerized tomography in the evaluation of laryngeal injury. Laryngoscope 101:202-207

11. Yen K, Vock P, Christe A, Scheurer E, Plattner T, Schön C, Aghayev E, Jackowski C, Beutler V, Thali MJ, Dirnhofer R (2007) Clinical forensic radiology in strangulation victims: forensic expertise based on magnetic resonance imaging (MRI) findings. Int J Legal Med 121(2):115-123, Epub 2007

12. Maxeiner H, Bockholdt B (2003) Homicidal and suicidal ligature strangulation - a comparison of the postmortem findings. Forensic Sci Int $137: 60-66$

13. Maxeiner H (1989) Morphologic findings for airway occlusion in strangulation (in German). Arch Kriminol 183:37-44

14. Spitz WU, Fisher RS (1993) Medicolegal investigation of death, 3rd edn. Charles C. Thomas, Springfield, IL, $829 \mathrm{p}$

15. Knight B (1991) Fatal pressure on the neck. Forensic pathology. Edwart Arnold, London/Melbourne/Auckland, pp 334-359

16. Jackowski C, Thali M, Aghayev E, Yen $\mathrm{K}$, Sonnenschein M, Zwygart K, Dirnhofer R, Vock P (2005) Postmortem imaging of blood and ist characteristics using MSCT and MRI Int J Legal Med 19:1-8
17. Bradley WG (1999) Hemorrhage. In: Stark DD, Bradley WG (eds) Magnetic resonance imaging. Mosby, St. Louis, pp 1329-1359

18. Cohen JA (1960) Coefficient of agreement for nominal scales. Educ Psychol Meas 20:37-46

19. Altman DG (1991) Practical statistics for medical research. Chapman and Hall, London

20. Thali MJ, Yen K, Schweitzer W, Vock P, Boesch C, Ozdoba C, Schroth G, Ith $\mathrm{M}$, Sonnenschein M, Doernhoefer T, Scheurer E, Plattner T, Dirnhofer R (2003) Virtopsy, a new imaging horizon in forensic pathology: virtual autopsy by postmortem multislice computed tomography (MSCT) and magnetic resonance imaging (MRI) - a feasibility study. J Forensic Sci 48(2):386-403

21. Thali MJ, Braun M, Buck U et al (2005) VIRTOPSY - scientific documentation, reconstruction and animation in forensic: individual and real 3D data based geo-metric approach including optical body/object surface and radiological CT/MRI scanning. J Forensic Sci 50:428-442 